\title{
P-1014/AD-0141
}

\section{Stream : Education and Integrated Care \\ Topic : Diabetes education \\ Impact of diabetes education workshop on improving knowledge, attitude and practices; interventional study in nurses}

K. Gupta ${ }^{1}$, S. Gupta ${ }^{2}$, S. Gathe ${ }^{3}$, S. Ugemuge ${ }^{4}$,S. Gupta ${ }^{5}$ A. Ambade ${ }^{6}$.

'Sunil's Diabetes Care n' Research Centre Pvt Ltd. Dietetics \& Therapeutic Diabetes Education, Nagpur, India.

Sunil's Diabetes Care n' Research Centre Pvt Ltd. 'Diabetology, ${ }^{, 4}$ Clinical Research, 'Opthalmology

${ }^{5}$ NKP Salve Medical College, Nagpur, India.

\section{Background:}

Nurses are considered as best educators, counselor to patients as they are involved in patient care closely especially for chronic diseases like Diabetes Mellitus. To become a good educator one should have good knowledge about the ailment. Previous data shows nurses from various health care settings lack sufficient knowledge about diabetes and diabetes management. Experiences shows better understanding about evidence-based practices, significantly influence disease outcome.

\section{AIM:}

Our study aimed to assess the knowledge, attitude and practices about diabetes amongst nurses through a pilot tested questionnaire and to evaluate the impact of structured multisession diabetes education workshop in improving the above characteristics.

\section{Methods:-}

Total 820 nurses from various hospitals and nursing colleges were invited to attend the diabetes workshop, which was organized at a public utility hall at Nagpur, India in November 2016 on occasion of World Diabetes Day 2016. Amongst them, 505 attended the session. A well designed 6 hour structured, multisession education workshop "Hello Diabetes" was conducted through lectures, demonstrations and role-plays focusing various aspects of diabetes. A pilot tested questionnaire was administered (pre \& post program) to evaluate knowledge, attitude and practices among them. From those attendees, $317(63 \%)$ nurses completed the pretest questionnaire appropriately. The same questionnaire was administered as the post-test. In all, 217 (43\%) completed both pretest and post-test precisely

The analysis of pre versus posttest scores in knowledge, attitude and practices was done using SPSS software 18. Association of significance was obtained with Wilcoxon Sign Rank Test for all the three categories Knowledge, Attitude and Practices.

\section{Results:}

- The median pre and post-test scores considering all questions were 63.33 and 66.67 respectively. The difference between these median scores was statistically significantly different than zero, as indicated by $\mathrm{P}$-value $<0.0001$ (Table1)

There were 16 questions pertaining to knowledge of the subject, which was analyzed independently. The pre- and post- median scores on these questions were 62.50 and 68.75 . The difference in the median scores was statistically significantly different than zero as indicated by P-value $<0.0001$ (Table2).

There were 10 questions related to attitude of nurses. The median score on attitudinal questions before training was 60 , while post-training was 70 . The difference of medians was statistically significantly different than zero as indicated by P-value $<0.0001$ (Table3).

There were 4 questions related to practice. The median score on these questions before training was 50 , while post-training was 75 . The difference between the pre and post-scores was statistically significantly different than zero as indicated by P-value $<0.0001$ (Table 4 )

\section{Discussion:-}

This study assessed present knowledge, attitude and practices about diabetes among nurses. There was a large knowledge gap regarding diabetes as demonstrated in this study as well as attitude and practices regarding diabetes. The diabetes education lectures "Hello Diabetes" had a significan impact on nurse's approach towards diabetes as shown in results. The role of structured education lecture is essential to imbibe knowledge of chronic ailment such as diabetes. Promoting continuing education workshop in diabetes for nurses requires continuous effort and creativity. Healthcare system administrators must acknowledge and prioritize the need for this education. The findings suggest that, in addition to educational programs, more focused workshops concerning diabetes care is needed to improve the care of patients with diabetes in long-term care facilities.

\section{Tables \& Graphs:}

Table 1: Comparison between pre and post test scores based on all questions

\begin{tabular}{|l|l|l|l|l|}
\hline Test & Mean & SD & Median & \multirow{2}{*}{ P-Value $^{*}$} \\
\hline Pre- & 62.30 & 9.70 & 63.33 & \multirow{2}{*}{$<0.0001(\mathrm{HS})$} \\
\hline Post- & 67.38 & 11.08 & 66.67 & \\
\hline
\end{tabular}

*Obtained using Wilcoxon signed rank test; HS: Highly Significant

The median difference between the overall pre and post-test scores was statistically significantly different than zero as obtained by Wilcoxon signed rank test.

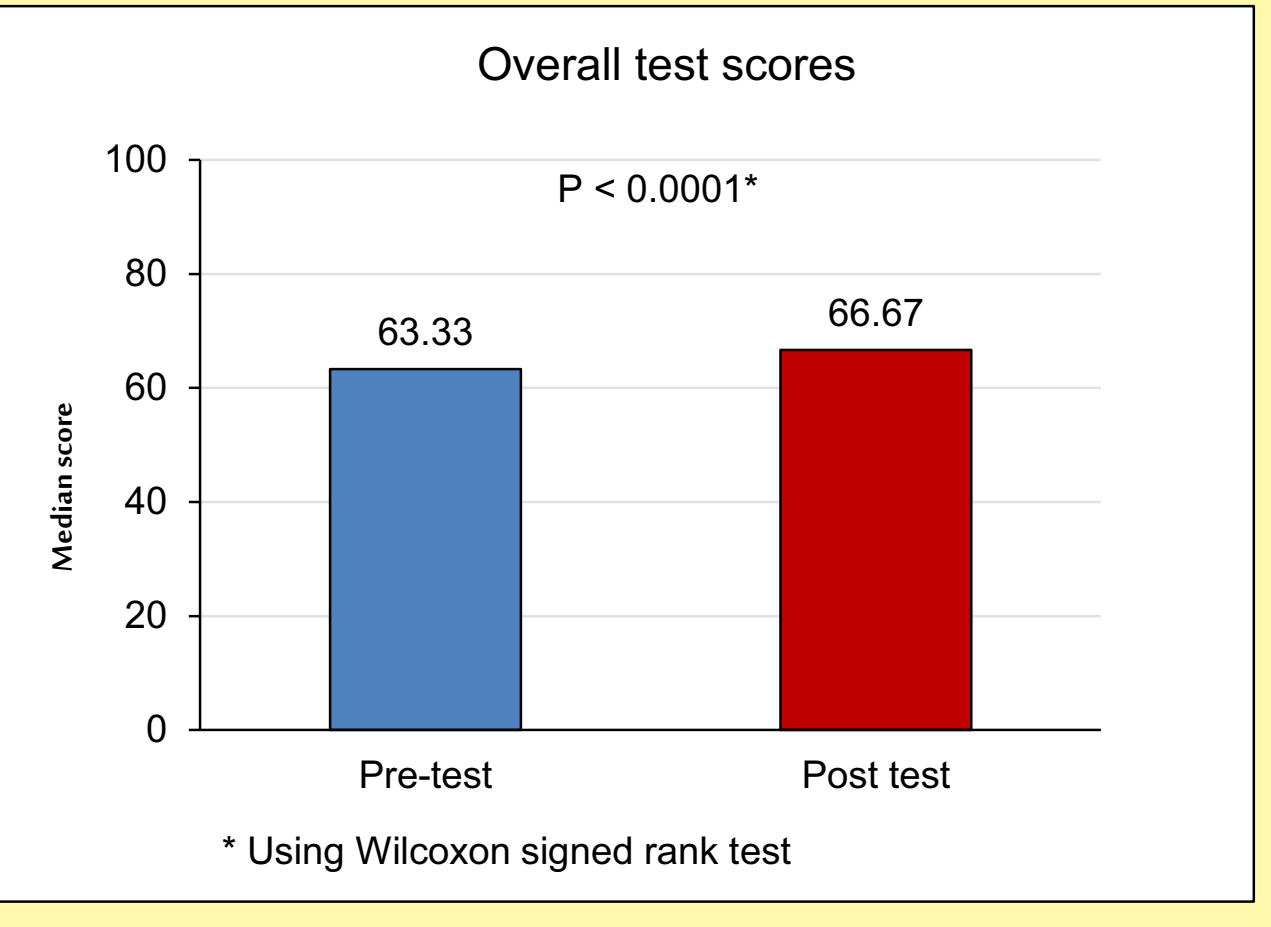

Figure 1: Bar chart showing mean score according to pre and post test for knowledge questions

Table 2: Comparison between pre- and post-test scores based on knowledge related questions

\begin{tabular}{|l|l|l|l|l|}
\hline Test & Mean & SD & Median & \multirow{2}{*}{ P-Value $^{*}$} \\
\hline Pre- & 63.76 & 11.04 & 65.50 & \multirow{2}{*}{$<0.0001(\mathrm{HS})$} \\
\hline Post- & 67.61 & 12.07 & 68.75 & \\
\hline
\end{tabular}

*Obtained using Wilcoxon signed rank test; HS: Highly Significant; †: Knowledge related questions

The median difference between the knowledge related pre- and post-test scores was statistically significantly different than zero as obtained by Wilcoxon signed rank test.

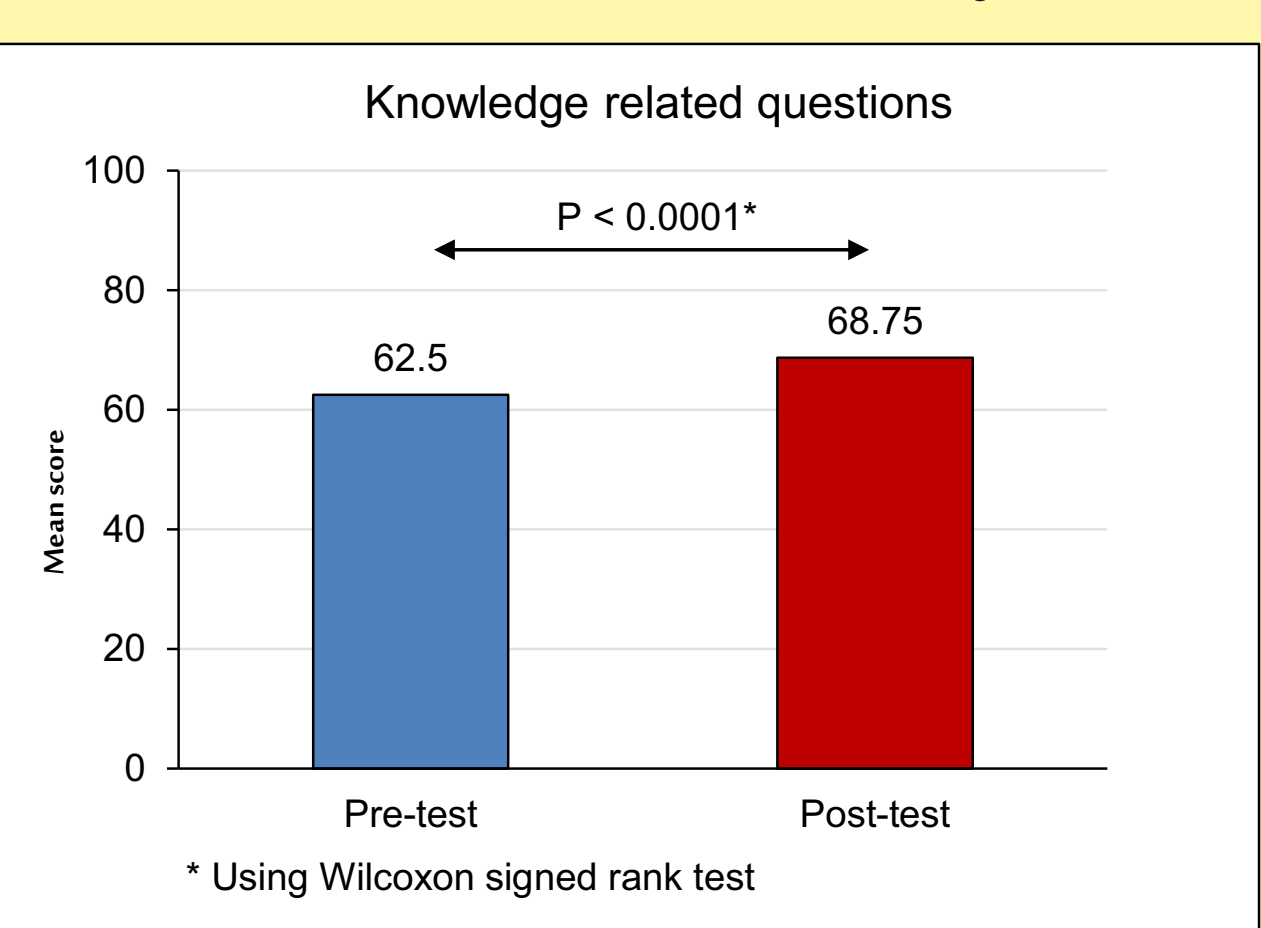

Figure 2: Bar chart showing mean score according to pre and post test for knowledge questions

Table 3: Comparison between pre- and post-test scores based on attitude related questions

\begin{tabular}{|l|l|l|l|l|}
\hline Test & Mean & SD & Median & \multirow{2}{*}{ P-Value $^{*}$} \\
\hline Pre- & 59.09 & 16.31 & 60.00 & \multirow{2}{*}{$<0.0001$ (HS) } \\
\hline Post- & 67.35 & 17.79 & 70.00 & \\
\hline
\end{tabular}

*Obtained using Wilcoxon signed rank test; HS: Highly Significant; †: Attituderelated questions

The median difference between the attitude related pre- and post-test scores was statistically significantly different than zero as obtained by Wilcoxon signed rank test.

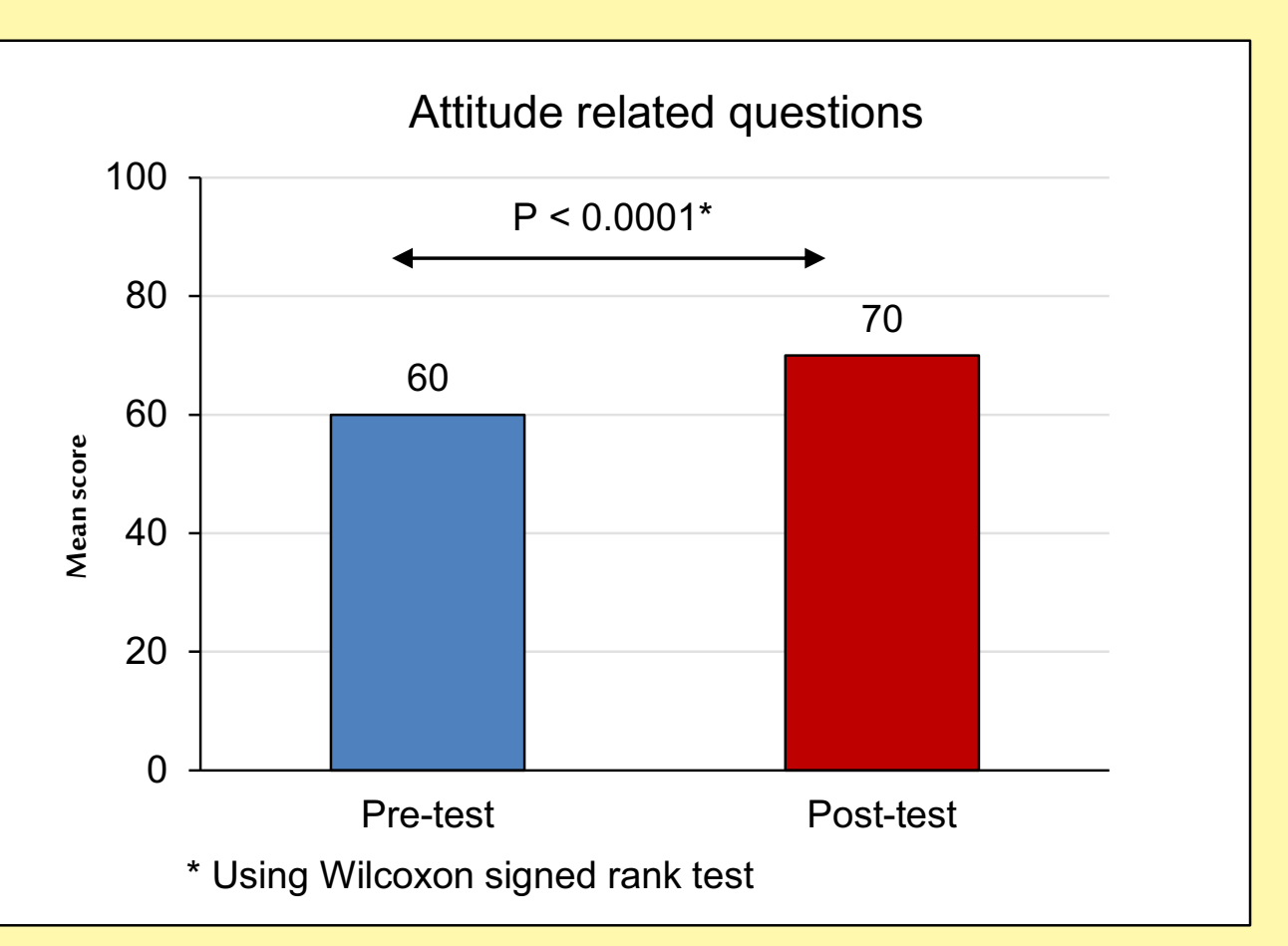

Figure 3: Bar chart showing mean score according to pre and post test for attitude questions

Table 4: Comparison between pre- and post-test scores based on practice related questions

\begin{tabular}{|l|l|l|l|l|}
\hline Test & Mean & SD & Median & \multirow{2}{*}{ P-Value $^{*}$} \\
\hline Pre- & 60.27 & 19.53 & 50.00 & \multirow{2}{*}{$<0.0001(\mathrm{HS})$} \\
\hline Post- & 66.55 & 18.47 & 75.00 & \\
\hline
\end{tabular}

*Obtained using Wilcoxon signed rank test; S: Significant; $\uparrow$ : Practice related questions

The median difference between the practice related pre- and post-test scores was statistically significantly different than zero as obtained by Wilcoxon signed rank test.

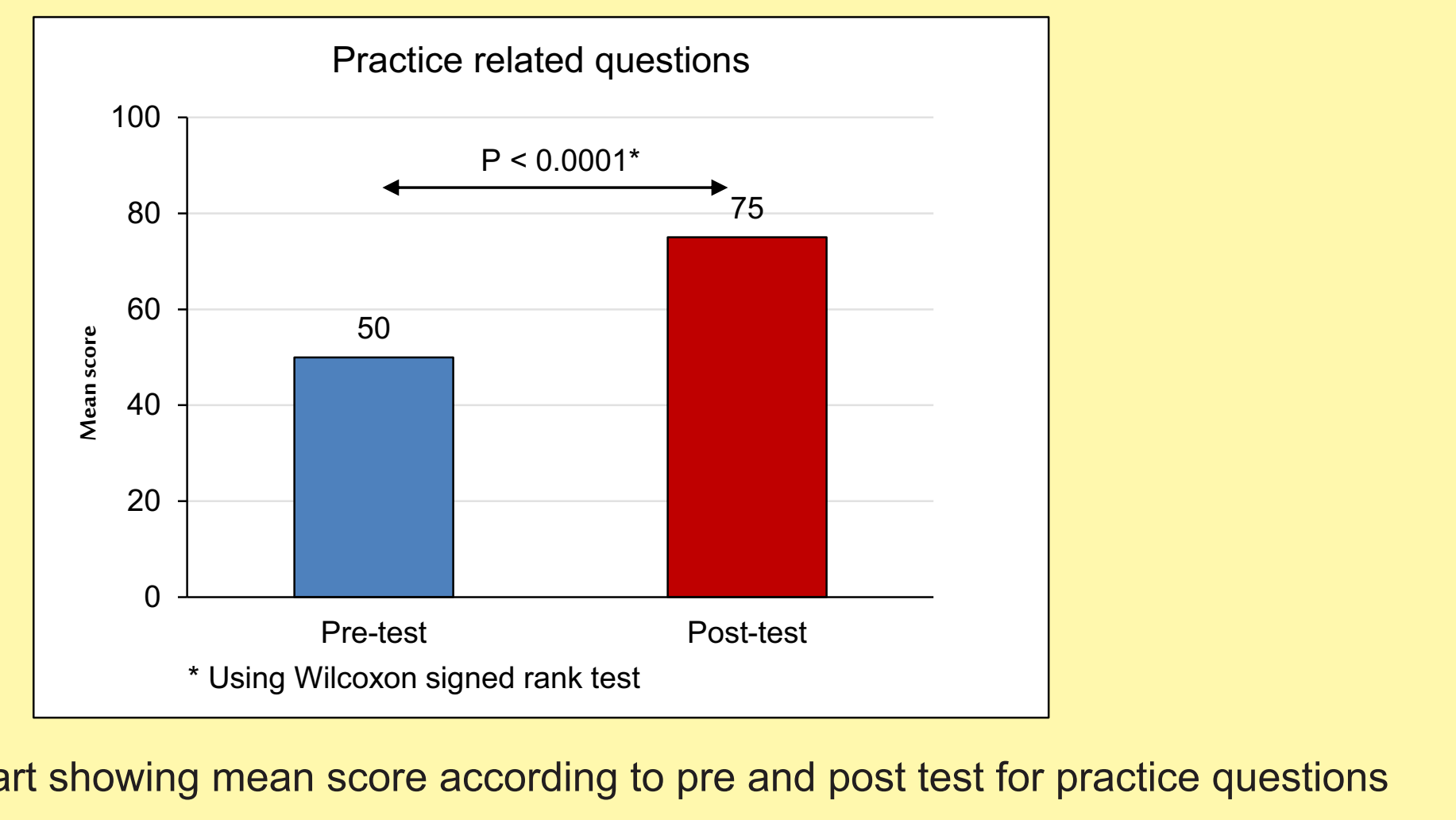

